\title{
A New Optimized Stochastic Approach for Multiple Integrals in Option Pricing
}

\author{
Venelin Todorov \\ Bulgarian Academy of Sciences \\ Institute of Mathematics and Informatics \\ ul. G. Bonchev 8, 1113 Sofia, Bulgaria \\ Bulgarian Academy of Sciences \\ Institute of Information and Communication Technologies \\ ul. G. Bonchev 25A, 1113 Sofia, Bulgaria \\ Email: vtodorov@math.bas.bg,venelin@parallel.bas.bg

\section{Stefka Fidanova \\ Bulgarian Academy of Sciences} \\ Institute of Information and Communication Technologies \\ ul. G. Bonchev 25A, 1113 Sofia, Bulgaria \\ Email: stefka@parallel.bas.bg
}

\begin{abstract}
In the present paper we evaluate European style options with an exponential payoff function with an optimized lattice rule based on a new optimal generating vector. A brief introduction of the theory of lattice rule has been given. We compare the performance of the new stochastic approach with a new optimal generating vector for multiple integrals up to 50 dimensions.
\end{abstract}

\section{INTRODUCTION}

Computational finance is one of the most important topic nowadays [4]. Nowadays Monte Carlo (MC) and quasi-Monte Carlo (QMC) methods have become a popular computational device for problems in finance [8]. The field of mathematical finance is becoming more sophisticated and quantitative and the scope of its applications is growing. The QMC methods using special deterministic sequences achieve higher accuracy and computational efficiency compared to the MC methods. Applications of low discrepancy sequences to financial problems are studied in [3]. Options have been widely traded since the creation of the organized exchange in 1973. The famous Black-Scholes model provides explicit closed form solutions for the values of the European style call and put options. Besides the applications in option pricing, Monte Carlo methods are also used in other problems in finance [2] and other branches of science.

Venelin Todorov is supported by the National Scientific Program "Young scientists and Postdoctoral candidates" 2020-2021. Venelin Todorov and Stoayn Poryazov are partially supported by Task 1.2 .5 of National Scientific Program "Information and Communication Technologies for a Single Digital Market in Science, Education and Security (ICT in SES)", contract No DO1-205/23.11.2018, financed by the Ministry of Education and Science in Bulgaria. The work is also supported by the Bulgarian National Science Fund under Project DN 12/5-2017 "Efficient Stochastic Methods and Algorithms for Large-Scale Problems".

\author{
Ivan Dimov \\ Bulgarian Academy of Sciences \\ Institute of Information and Communication Technologies \\ ul. G. Bonchev 25A, 1113 Sofia, Bulgaria \\ Email: ivdimov@bas.bg
}

\author{
Stoyan Poryazov \\ Bulgarian Academy of Sciences \\ Institute of Mathematics and Informatics \\ ul. G. Bonchev 8, 1113 Sofia, Bulgaria \\ Bulgarian Academy of Sciences \\ Email: stoyan@math.bas.bg
}

Definition 1: A European call (put) option is a contract which gives its holder a right, but not an obligation, to buy (sell) predefined quantity of underlying asset $S$ at a prescribed time $T$ (expiry date) at a prescribed exercise (strike) price $E$. An American call (put) option is the same as the European call (put), except that the American options can be exercised at any time during their lifetime, not just at expiry.

The definitions of the risk neutrality and the risk-free interest rate $r$ can be found in [8].

\section{DESCRIPTION OF THE OPTION PRICING PROBLEM}

The value of an asset $S$ as a function of time is modeled by the stochastic differential equation

$$
\mathrm{d} S=\mu S \mathrm{~d} t+\sigma S \mathrm{~d} X
$$

where $\sigma$ is the volatility of the asset, characterizing the fluctuations in the price, $\mu$ is the drift rate, which is a measure of the average rate of growth of the asset price and $\mathrm{d} X$ is the increment of a standard Wiener process. The wellknown Black-Scholes (BS) model for European call options is described with the following partial differential equation

$$
\frac{\partial V}{\partial t}+\frac{1}{2} \sigma^{2} S^{2} \frac{\partial^{2} V}{\partial S^{2}}+r S \frac{\partial V}{\partial S}-r V=0
$$

where $r$ is the risk-free interest rate. The value $V(S, t)$ of the call option satisfies the final condition at the time of expiry $V(S, T)=\max (S-E, 0)$, where $E$ is the strike price. The boundary conditions of a Dirichlet type are as follows:

$$
V(0, t)=0, \quad V(S, t) \sim S-E e^{-r(T-t)}, S \rightarrow \infty .
$$

The European put options satisfy the same BS equation (2) with final and boundary conditions

$$
V(S, T)=\max (E-S, 0),
$$




$$
V(0, t)=E e^{-r(T-t)}, \quad V(S, t) \sim 0, S \rightarrow \infty .
$$

The analytical solution of the BS equation for call options is expressed in an explicit closed form as

$$
V(S, t)=C(S, t)=S \mathrm{~N}\left(d_{1}\right)-E e^{-r(T-t)} \mathrm{N}\left(d_{2}\right),
$$

where

$$
\begin{aligned}
& d_{1}=\frac{\ln (S / E)+\left(r+\sigma^{2} / 2\right)(T-t)}{\sigma \sqrt{T-t}}, \\
& d_{2}=\frac{\ln (S / E)+\left(r-\sigma^{2} / 2\right)(T-t)}{\sigma \sqrt{T-t}},
\end{aligned}
$$

and $\mathrm{N}(z)$ is the cumulative distribution function of the standard normal distribution. Similarly, the European put options price is computed with the formula

$$
V(S, t)=P(S, t)=E e^{-r(T-t)} \mathrm{N}\left(-d_{2}\right)-S \mathrm{~N}\left(-d_{1}\right) .
$$

MC and QMC methods are suitable when the solution is expressed as the expectation of a random variable, which is the case of the risk-neutral evaluation formula for the European options [8]:

$$
V(S, t)=E\left(e^{-r(T-t)} h(S(T)) \mid S(t)=S, \mu=r\right),
$$

where $E$ is the expectation operator and $h(S)$ is the payoff function. The payoff function of the European option satisfies

$$
h(S)=\max (S-E, 0)
$$

for the call option and

$$
h(S)=\max (E-S, 0)
$$

for the put option.

Let's deal with a European call option whose payoff depends on $k>1$ assets with prices $S_{i}, i=1, \ldots, k$. Each asset is modeled by a random walk (1)

$$
\mathrm{d} S_{i}=\mu_{i} S_{i} \mathrm{~d} t+\sigma_{i} S_{i} \mathrm{~d} X_{i}
$$

where $\sigma_{i}$ is the annualized standard deviation for the $i$-th asset and $\mathrm{d} X_{i}$ is an increment of a Brownian motion. The Brownian motion is a special type Markov stochastic process, which has the property $\mathrm{d} X \sim \mathrm{N}(0, \sqrt{d t})$, where $\mathrm{N}(\mu, \sigma)$ is the normal distribution with mean $\mu$ and variance $\sigma^{2}$.

Now suppose that at expiry time $T$, the payoff is given by $h\left(S_{1}^{\prime}, \ldots, S_{k}^{\prime}\right)$, where $S^{\prime}$ denotes the value of the $i$-th asset at expiry. Assuming risk neutrality, the value of the option satisfies

$$
\begin{gathered}
V=e^{-r(T-t)}(2 \pi(T-t))^{-k / 2}(\operatorname{det} \Sigma)^{-1 / 2}\left(\sigma_{1} \ldots \sigma_{k}\right)^{-1} \\
\int_{0}^{\infty} \ldots \int_{0}^{\infty} \frac{h\left(S_{1}^{\prime}, \ldots, S_{k}^{\prime}\right)}{S_{1}^{\prime} \ldots S_{k}^{\prime}} \\
\exp \left(-0.5 \alpha^{\top} \Sigma^{-1} \alpha\right) \mathrm{d} S_{1}^{\prime} \ldots \mathrm{d} S_{k}^{\prime}
\end{gathered}
$$

where

$$
\alpha_{i}=\left(\sigma_{i}(T-t)^{1 / 2}\right)^{-1}\left(\ln \left(S_{i}^{\prime} / S_{i}\right)-\left(r-\sigma_{i}^{2} / 2\right)(T-t)\right),
$$

$r$ is the risk-free interest rate and $\Sigma$ is the covariance matrix, whose entry with index $(i, j)$ is the covariance $\mathrm{d} X_{i}$ and $\mathrm{d} X_{j}$ of the $k$ assets. We will consider the special case when the payoff function is the exponent function which is important for some application in finance, see [8].

\section{DESCRIPTION OF THE NEW OPTIMIZED LATTICE RULE}

Consider the quadrature formula

$$
I_{N}(f)=\frac{1}{N} \sum_{i=0}^{N-1} f\left(x_{i}\right)
$$

where $P_{N}=\left\{x_{0}, x_{1}, \ldots, x_{N-1}\right\}, x_{i} \in[0,1)^{s}$ are the integration nodes of the formula.

The integration nodes, of the lattice rules proposed by Korobov [6], are defined by the following formula:

$$
x_{k}=\left(\left\{\frac{k z_{1}}{N}\right\},\left\{\frac{k z_{2}}{N}\right\}, \ldots,\left\{\frac{k z_{s}}{N}\right\}\right), k=1,2, \ldots, N
$$

where $N$ is the number of the nodes, $z$ is an $s$-dimensional generating vector of the lattice set and $\{a\}=a-[a]$ is the fractional part of $a$. The lattice rules with nodes (4) and generators $z$ are called "rank 1" rules. The class $E_{s}^{\alpha}(c)$ is defined by Koborov.

Definition 2: ([6]) We say that $f(x)$ belongs to the class of functions $E_{s}^{\alpha}(c)$ for $\alpha>1$ and $c>0$, if $f$ is a periodic function with period 1 for every of its components $x_{i}, i=$ $1,2 \ldots, s$, defined over the unit cube $[0,1]^{s}$ and its coefficients satisfy the following inequalities:

$$
|a(m)|<\frac{c}{\left(\bar{m}_{1} \ldots \bar{m}_{s}\right)^{\alpha}},
$$

where

$$
\bar{m}= \begin{cases}|m|, & |m| \neq 0 \\ 0, & m=0\end{cases}
$$

and the constant $c$ does not depend on $m_{1}, \ldots, m_{s}$.

Theorem 1: ([1]) There exists an optimal choice of the generating vector $z$, for which the error of integration satisfies

$$
\left|\frac{1}{N} \sum_{k=0}^{N-1} f\left(\left\{\frac{k}{N} z\right\}\right)-\int_{[0,1)^{s}} f(u) d u\right| \leq c d(s, \alpha) \frac{(\log N)^{\beta(s, \alpha)}}{N^{\alpha}},
$$

for the function $f \in E_{s}^{\alpha}(c)$, where $\alpha>1$ and $d(s, \alpha), \beta(s, \alpha)$ do not depend on $N$. Moreover, if $N$ is a prime number, then $\beta(s, \alpha)=\alpha(s-1)$.

The generating vector $z$, for which inequality (5) is satisfied, is an optimal generating vector in the sense of Korobov. The search over all possible $s$-dimensional lattice points $z \bmod n$, requires a significant computational effort in high dimensions. Thus, it is desirable to have efficient search schemes for finding the good lattice points. 
A. An optimized lattice rule based on an optimal generating vectors

For a given number of lattice points $N$, the performance of the routine is affected by the choice of the generator vector $z$. In 1981 Hua and Wang [5] generalized the Fibonacci numbers for any dimension. We refer to the lattice method with a generating vector

$$
z=\left(1, F_{l+1}^{(s)}, \ldots, F_{l+s-1}^{(s)}\right), \quad n_{l}=F_{l}^{(s)},
$$

where $F_{j}^{(s)}$ is the generalized Fibonacci number of dimension $s$, as Fibonacci lattice rule (FIBO). The advantages of the FIBO method are the linear complexity of the algorithm and fast computation of the generating vector. We propose an optimized lattice rule with a specific choice of the optimal 200-generating vector, constructed by Dirk Nuyens [7] in 2016 and the computation involves fast component by component operations. The method is improved by generating the points from a lattice sequence in base 2 in gray coded radical inverse ordering. The algorithm for reversing the digits of an integer number is computationally efficient. This optimal generating vector has been applied for the first time to a problem is computational finance and it gives superior results to the stochastic approaches used up to now. Since the performance of a lattice rule depends on the choice of the generator vectors, the 200-dimensional optimal vector is an optimization over the Fibonacci generalized vector (6).

\section{NUMERICAL EXAMPLES AND RESULTS}

The experimental results include the evaluation of the multidimensional integrals

$$
I_{s}=\int_{[0,1]^{s}} \exp \left(\prod_{i=1}^{s} x_{i}\right) d x_{1} \ldots d x_{s}
$$

By expanding the exponential function in Taylor series and integrating the terms $\left(x_{1} \cdots x_{s}\right)^{n}$ we obtain

$$
\begin{gathered}
\int_{[0,1]^{s}} \exp \left(\prod_{i=1}^{s} x_{i}\right)= \\
=\sum_{n=0}^{\infty} \frac{1}{(n+1)^{s} n !}={ }_{s} F_{s}(1, \cdots, 1 ; 2, \cdots, 2 ; 1),
\end{gathered}
$$

where ${ }_{p} F_{q}\left(a_{1}, \cdots, a_{p} ; b_{1}, \cdots, b_{q} ; x\right)$ is the generalized hypergeometric function

$$
{ }_{p} F_{q}\left(a_{1}, \cdots, a_{p} ; b_{1}, \cdots, b_{q} ; x\right)=\sum_{n=0}^{\infty} \frac{\left(a_{1}\right)_{n} \cdots\left(a_{p}\right)_{n}}{\left(b_{1}\right)_{n} \cdots\left(b_{q}\right)_{n}} \frac{x^{n}}{n !},
$$

and $(c)_{n}=c(c+1) \cdots(c+n-1)$ is the Pochhammer symbol.

$$
\begin{gathered}
\int_{[0,1]^{3}} \exp \left(x_{1} x_{2} x_{3}\right) \approx 1.14649907 . \\
\int_{[0,1]^{5}} \exp \left(\sum_{i=1}^{5} 0.5 a_{i} x_{i}^{2}\left(2+\sin \sum_{j=1, j \neq i}^{5} x_{j}\right)\right) \approx 2.923651,
\end{gathered}
$$

where $a_{i}=(1,0.5,0.2,0.2,0.2)$

$$
\begin{gathered}
\int_{[0,1]^{8}} \exp \left(\sum_{i=1}^{8} 0.1 x_{i}\right)=1.496805 . \\
\int_{[0,1]^{20}} \exp \left(\prod_{i=1}^{20} x_{i}\right) \approx 1.00000949634 .
\end{gathered}
$$

We also compare the performance of the methods on a 50 dimensional integral:

$$
I_{50}=\int_{[0,1]^{50}} \exp \left(\prod_{i=1}^{50} x_{i}\right)
$$

We evaluate his reference value by expanding the exponential function in Taylor series and integrating the terms $\left(x_{1} \cdots x_{50}\right)^{n}$ we obtain

$$
\begin{gathered}
\int_{[0,1]^{50}} \exp \left(\prod_{i=1}^{50} x_{i}\right)= \\
=\sum_{n=0}^{\infty} \frac{1}{(n+1)^{50} n !}={ }_{50} F_{50}(1, \cdots, 1 ; 2, \cdots, 2 ; 1),
\end{gathered}
$$

where ${ }_{p} F_{q}\left(a_{1}, \cdots, a_{p} ; b_{1}, \cdots, b_{q} ; x\right)$ is the generalized hypergeometric function

$$
{ }_{p} F_{q}\left(a_{1}, \cdots, a_{p} ; b_{1}, \cdots, b_{q} ; x\right)=\sum_{n=0}^{\infty} \frac{\left(a_{1}\right)_{n} \cdots\left(a_{p}\right)_{n}}{\left(b_{1}\right)_{n} \cdots\left(b_{q}\right)_{n}} \frac{x^{n}}{n !},
$$

and $(c)_{n}=c(c+1) \cdots(c+n-1)$ is the Pochhammer symbol.

The experimental results are presented in the Tables below. Each table includes the relative error of the QMC method, the CPU-time and the number of integration nodes. We compare the performance of the optimized lattice rule (OPT), the Fibonacci based lattice rule (FIBO) and two other stochastic approaches Adapt for the wellknown adaptive stochastic approach and Sobol for the well know Sobol quasi-random sequence.

Table I

THE RELATIVE ERROR FOR 3 DIMENSIONAL INTEGRAL

\begin{tabular}{|c|c|c|c|c|c|c|c|c|}
\hline $\mathrm{N}$ & OPT & time & Adapt & time & FIBO & time & Sobol & time \\
\hline 19513 & $1.93 \mathrm{e}-5$ & 0.01 & $3.21 \mathrm{e}-4$ & 2.21 & $4.69 \mathrm{e}-4$ & 0.02 & $4.98 \mathrm{e}-5$ & 0.56 \\
35890 & $3.18 \mathrm{e}-6$ & 0.04 & $6.55 \mathrm{e}-5$ & 6.41 & $5.46 \mathrm{e}-6$ & 0.06 & $1.56 \mathrm{e}-5$ & 1.45 \\
66012 & $2.65 \mathrm{e}-6$ & 0.07 & $5.12 \mathrm{e}-5$ & 9.86 & $5.34 \mathrm{e}-6$ & 0.11 & $8.11 \mathrm{e}-6$ & 2.31 \\
121415 & $9.16 \mathrm{e}-7$ & 0.12 & $5.11 \mathrm{e}-5$ & 15.4 & $5.34 \mathrm{e}-6$ & 0.12 & $3.08 \mathrm{e}-6$ & 3.80 \\
223317 & $8.01 \mathrm{e}-7$ & 0.20 & $9.34 \mathrm{e}-5$ & 24.2 & $1.73 \mathrm{e}-6$ & 0.22 & $2.05 \mathrm{e}-6$ & 6.13 \\
\hline
\end{tabular}

Table II

THE RELATIVE ERROR FOR 3 DIMENSIONAL INTEGRAL

\begin{tabular}{|c|c|c|c|c|}
\hline time,s & OPT & Adapt & FIBO & sobol \\
\hline 0.1 & $9.16 \mathrm{e}-7$ & $8.67 \mathrm{e}-4$ & $1.32 \mathrm{e}-6$ & $3.21 \mathrm{e}-4$ \\
1 & $6.37 \mathrm{e}-7$ & $2.96 \mathrm{e}-5$ & $3.22 \mathrm{e}-7$ & $8.21 \mathrm{e}-5$ \\
2 & $4.22 \mathrm{e}-7$ & $5.45 \mathrm{e}-4$ & $2.06 \mathrm{e}-7$ & $2.96 \mathrm{e}-5$ \\
5 & $1.84 \mathrm{e}-7$ & $1.14 \mathrm{e}-4$ & $1.47 \mathrm{e}-7$ & $5.00 \mathrm{e}-6$ \\
10 & $6.09 \mathrm{e}-8$ & $6.56 \mathrm{e}-5$ & $3.89 \mathrm{e}-7$ & $2.71 \mathrm{e}-6$ \\
20 & $1.57 \mathrm{e}-8$ & $2.04 \mathrm{e}-5$ & $1.53 \mathrm{e}-8$ & $1.88 \mathrm{e}-6$ \\
\hline
\end{tabular}

The Adaptive Monte Carlo gives the worst results, because its strength is when the integrand has some pecularities. Sobol is suitable for non-smooth functions and it is outperformed by the other methods in low dimensions (Tables I,II,III,IV). The accuracy 
Table III

THE RELATIVE ERROR FOR 5 DIMENSIONAL INTEGRAL

\begin{tabular}{|c|c|c|c|c|c|c|c|c|}
\hline $\mathrm{N}$ & OPT & time & Adapt & time & FIBO & time & Sobol & time \\
\hline 13624 & $6.72 \mathrm{e}-5$ & 0.02 & $1.89 \mathrm{e}-3$ & 2.33 & $9.59 \mathrm{e}-4$ & 0.03 & $1.76 \mathrm{e}-4$ & 0.56 \\
52656 & $1.53 \mathrm{e}-5$ & 0.06 & $2.31 \mathrm{e}-3$ & 6.18 & $6.96 \mathrm{e}-4$ & 0.06 & $5.05 \mathrm{e}-5$ & 1.45 \\
103519 & $8.48 \mathrm{e}-6$ & 0.09 & $2.01 \mathrm{e}-3$ & 9.94 & $8.72 \mathrm{e}-5$ & 0.13 & $2.70 \mathrm{e}-5$ & 2.52 \\
203513 & $6.25 \mathrm{e}-6$ & 0.15 & $3.42 \mathrm{e}-4$ & 16.2 & $8.04 \mathrm{e}-5$ & 0.25 & $7.57 \mathrm{e}-6$ & 6.07 \\
400096 & $8.16 \mathrm{e}-7$ & 0.40 & $9.12 \mathrm{e}-4$ & 45.6 & $7.26 \mathrm{e}-5$ & 0.50 & $2.52 \mathrm{e}-6$ & 10.63 \\
\hline
\end{tabular}

Table IV

TIMES FOR 5 DIMENSIONAL INTEGRAL

\begin{tabular}{|c|c|c|c|c|}
\hline time,s & OPT & Adapt & FIBO & sobol \\
\hline 0.1 & $3.07 \mathrm{e}-6$ & $1.34 \mathrm{e}-2$ & $7.26 \mathrm{e}-5$ & $8.22 \mathrm{e}-4$ \\
1 & $1.32 \mathrm{e}-6$ & $2.44 \mathrm{e}-3$ & $2.28 \mathrm{e}-5$ & $2.91 \mathrm{e}-4$ \\
5 & $1.13 \mathrm{e}-6$ & $4.93 \mathrm{e}-4$ & $5.94 \mathrm{e}-6$ & $1.71 \mathrm{e}-5$ \\
10 & $5.47 \mathrm{e}-7$ & $1.88 \mathrm{e}-3$ & $3.85 \mathrm{e}-7$ & $1.79 \mathrm{e}-5$ \\
20 & $3.52 \mathrm{e}-7$ & $2.71 \mathrm{e}-4$ & $7.49 \mathrm{e}-7$ & $4.96 \mathrm{e}-6$ \\
\hline
\end{tabular}

Table V

THE RELATIVE ERROR FOR 8 DIMENSIONAL INTEGRAL

\begin{tabular}{|c|c|c|c|c|c|c|c|c|}
\hline $\mathrm{N}$ & OPT & time & Adapt & time & FIBO & time & Sobol & time \\
\hline 16128 & $1.79 \mathrm{e}-6$ & 0.04 & $1.10 \mathrm{e}-5$ & 12.6 & $8.08 \mathrm{e}-4$ & 0.03 & $8.87 \mathrm{e}-5$ & 0.13 \\
32192 & $1.56 \mathrm{e}-6$ & 0.05 & $3.32 \mathrm{e}-5$ & 33.3 & $1.03 \mathrm{e}-4$ & 0.07 & $5.42 \mathrm{e}-5$ & 0.58 \\
64256 & $8.01 \mathrm{e}-7$ & 0.08 & $4.65 \mathrm{e}-5$ & 54.2 & $5.03 \mathrm{e}-5$ & 0.11 & $2.34 \mathrm{e}-5$ & 2.49 \\
128257 & $6.22 \mathrm{e}-7$ & 0.13 & $8.25 \mathrm{e}-6$ & 88.3 & $8.13 \mathrm{e}-6$ & 0.14 & $4.45 \mathrm{e}-6$ & 6.36 \\
510994 & $3.21 \mathrm{e}-7$ & 0.34 & $7.07 \mathrm{e}-6$ & 233.6 & $5.95 \mathrm{e}-6$ & 0.57 & $3.32 \mathrm{e}-6$ & 19.45 \\
\hline
\end{tabular}

Table VI

TIMES FOR THE 8 DIMENSIONAL INTEGRAL

\begin{tabular}{|c|c|c|c|c|}
\hline time,s & OPT & Adapt & FIBO & sobol \\
\hline 1 & $2.18 \mathrm{e}-7$ & $6.34 \mathrm{e}-4$ & $5.34 \mathrm{e}-6$ & $2.02 \mathrm{e}-5$ \\
2 & $1.32 \mathrm{e}-7$ & $1.58 \mathrm{e}-4$ & $2.57 \mathrm{e}-6$ & $2.73 \mathrm{e}-5$ \\
5 & $9.03 \mathrm{e}-8$ & $1.44 \mathrm{e}-4$ & $1.52 \mathrm{e}-7$ & $8.88 \mathrm{e}-6$ \\
10 & $5.00 \mathrm{e}-8$ & $6.61 \mathrm{e}-5$ & $3.45 \mathrm{e}-6$ & $5.23 \mathrm{e}-6$ \\
20 & $2.55 \mathrm{e}-8$ & $2.77 \mathrm{e}-5$ & $1.82 \mathrm{e}-7$ & $2.11 \mathrm{e}-6$ \\
\hline
\end{tabular}

Table VII

THE RELATIVE ERROR FOR 20 DIMENSIONAL INTEGRAL

\begin{tabular}{|c|c|c|c|c|c|c|c|c|}
\hline $\mathrm{N}$ & OPT & time & Adapt & time & FIBO & time & Sobol & time \\
\hline 2048 & $2.84 \mathrm{e}-6$ & 0.02 & $1.14 \mathrm{e}-2$ & 8.6 & $8.22 \mathrm{e}-5$ & 0.03 & $8.44 \mathrm{e}-4$ & 0.13 \\
16384 & $1.04 \mathrm{e}-6$ & 0.12 & $4.96 \mathrm{e}-4$ & 60.3 & $3.12 \mathrm{e}-5$ & 0.13 & $6.82 \mathrm{e}-5$ & 1.68 \\
65536 & $9.21 \mathrm{e}-7$ & 0.91 & $9.75 \mathrm{e}-4$ & 474.2 & $1.36 \mathrm{e}-5$ & 1.17 & $8.34 \mathrm{e}-6$ & 8.69 \\
131072 & $6.15 \mathrm{e}-7$ & 2.13 & $1.25 \mathrm{e}-5$ & 888.3 & $8.85 \mathrm{e}-6$ & 2.34 & $3.77 \mathrm{e}-6$ & 14.36 \\
524288 & $5.33 \mathrm{e}-8$ & 8.13 & $1.96 \mathrm{e}-6$ & 2356 & $2.15 \mathrm{e}-6$ & 8.34 & $1.91 \mathrm{e}-7$ & 57 \\
\hline
\end{tabular}

Table VIII

TIMES FOR THE 20 DIMENSIONAL INTEGRAL

\begin{tabular}{|c|c|c|c|c|}
\hline time,s & OPT & Adapt & FIBO & sobol \\
\hline 1 & $9.14 \mathrm{e}-7$ & $1.58 \mathrm{e}-3$ & $1.48 \mathrm{e}-5$ & $3.25 \mathrm{e}-5$ \\
2 & $1.08 \mathrm{e}-7$ & $1.028 \mathrm{e}-3$ & $9.17 \mathrm{e}-6$ & $3.97 \mathrm{e}-5$ \\
5 & $5.87 \mathrm{e}-8$ & $8.58 \mathrm{e}-4$ & $5.19 \mathrm{e}-6$ & $1.45 \mathrm{e}-5$ \\
10 & $3.56 \mathrm{e}-8$ & $4.02 \mathrm{e}-4$ & $1.73 \mathrm{e}-6$ & $2.71 \mathrm{e}-6$ \\
20 & $1.23 \mathrm{e}-8$ & $1.13 \mathrm{e}-4$ & $1.38 \mathrm{e}-7$ & $1.76 \mathrm{e}-6$ \\
\hline
\end{tabular}

of the FIBO method and it has comparable execution time (Table 2). The Fibonacci lattice rule is the fastest method but its accuracy is lower than the accuracy of the lattice rules with increasing the dimensionality of the integral (Tables V,VI) and Sobol QMC becomes more accurate than FIBO (Tables VII,VIII). In highest dimension the optimized stochastic approach OPT has the lowest
Table IX

THE RELATIVE ERROR FOR 50 DIMENSIONAL INTEGRAL

\begin{tabular}{|c|c|c|c|c|c|c|}
\hline $\mathrm{N}$ & OPT & time & FIBO & time & Sobol & time \\
\hline $2^{10}$ & $2.88 \mathrm{e}-7$ & 0.05 & $6.23 \mathrm{e}-4$ & 0.08 & $8.88 \mathrm{e}-5$ & 3.5 \\
$2^{12}$ & $1.88 \mathrm{e}-7$ & 0.17 & $1.55 \mathrm{e}-4$ & 0.35 & $8.88 \mathrm{e}-5$ & 16 \\
$2^{16}$ & $5.44 \mathrm{e}-8$ & 2.14 & $9.72 \mathrm{e}-5$ & 5.21 & $8.88 \mathrm{e}-5$ & 73 \\
$2^{20}$ & $4.28 \mathrm{e}-8$ & 17.65 & $6.08 \mathrm{e}-5$ & 32.76 & $8.88 \mathrm{e}-6$ & 276 \\
\hline
\end{tabular}

Table X

TIMES FOR THE 50 DIMENSIONAL INTEGRAL

\begin{tabular}{|c|c|c|c|}
\hline time,s & OPT & FIBO & sobol \\
\hline 1 & $9.14 \mathrm{e}-8$ & $1.58 \mathrm{e}-3$ & $1.48 \mathrm{e}-5$ \\
2 & $3.68 \mathrm{e}-8$ & $1.028 \mathrm{e}-3$ & $9.17 \mathrm{e}-6$ \\
5 & $2.67 \mathrm{e}-8$ & $8.58 \mathrm{e}-4$ & $5.19 \mathrm{e}-6$ \\
10 & $3.34 \mathrm{e}-9$ & $4.02 \mathrm{e}-4$ & $1.73 \mathrm{e}-6$ \\
20 & $1.53 \mathrm{e}-9$ & $1.13 \mathrm{e}-4$ & $1.38 \mathrm{e}-7$ \\
\hline
\end{tabular}

relative error and a very high accuracy regardless of the dimension. (Table IX,X). The main advantage of the presented algorithms is their linear computational complexity with respect to dimension, while the deterministic methods suffer from the so-called curse of dimensionality and they become impractical in higher dimensions. The best performance of the optimized method is explained by the choice of the optimal generating vector, whose computation uses fast component-by-component operations. To summarize the optimized lattice rule gives the best results in terms of lowest relative errors and higher computational efficiency.

\section{CONCLUSION}

The applications of optimized lattice rules to the problem of option pricing is studied in the paper. The experimental results from Tables show that the optimized lattice rule with the special choice of the optimal generating vectors has the best performance with respect to relative error and computational time. The progress on the problem of option pricing and the computational finance area is closely related to the development of reliable algorithms for multidimensional numerical integration.

\section{REFERENCES}

[1] N. Bahvalov (1959) On the approximate calculation of multiple integrals, Journal of Complexity, Volume 31, Issue 4, 2015, Pages 502-516, ISSN 0885-064X, https://doi.org/10.1016/j.jco.2014.12.003.

[2] F. Black and M. Scholes (1973) The Pricing of Options and Corporate Liabilities, The Journal of Political Economy 81(3), 637-659, http://dx.doi.org/10.1086/260062.

[3] P.P. Boyle (1977) Options: a Monte Carlo Approach, Journal of Financial Economics 4(3), 323-338, https://doi.org/10.1016/0304405X(77)90005-8.

[4] Centeno, V., Georgiev, I. R., Mihova, V., \& Pavlov, V. (2019, October). Price forecasting and risk portfolio optimization. In AIP Conference Proceedings (Vol. 2164, No. 1, p. 060006). AIP Publishing LLC., https://doi.org/10.1063/1.5130808.

[5] L. K. Hua and Y. Wang, Applications of number theory to numerical analysis, (Springer 1981).

[6] N.M. Korobov, Number-theoretical methods in approximate analysis (Fizmatgiz, Moscow, 1963).

[7] Kuo, F.Y., Nuyens, D. Application of Quasi-Monte Carlo Methods to Elliptic PDEs with Random Diffusion Coefficients: A Survey of Analysis and Implementation. Found Comput Math 16, 16311696 (2016). https://doi.org/10.1007/s10208-016-9329-5.

[8] Lai Y., Spanier J. (2000) Applications of Monte Carlo/Quasi-Monte Carlo Methods in Finance: Option Pricing. In: Niederreiter H., Spanier J. (eds) Monte-Carlo and Quasi-Monte Carlo Methods 1998. Springer, Berlin, Heidelberg. https://doi.org/10.1007/978-3-642-59657-5_19 